EPJ Web of Conferences 70, 00049 (2014)

DOI: $10.1051 /$ epjconf/ 20147000049

(C) Owned by the authors, published by EDP Sciences, 2014

\title{
Indirect Dark Matter search with the ANTARES Deep-Sea Cherenkov detector
}

\author{
Paolo Fermani ${ }^{1,2, a}$ on behalf of the ANTARES collaboration \\ 1 "Sapienza" Università di Roma, Dipartimento di Fisica, P.le Aldo Moro 5, 00185, Roma, Italy \\ ${ }^{2}$ INFN Roma, P.le Aldo Moro 2, 00185, Roma, Italy
}

\begin{abstract}
In 2008 the ANTARES collaboration completed the construction of an underwater neutrino telescope in the Mediterranean Sea, located $40 \mathrm{~km}$ off the French coast at a depth of $2475 \mathrm{~m}$. With an effective area for upward muon detection of about 0.05 $\mathrm{km}^{2}$, depending on neutrino energy, ANTARES is the largest neutrino detector currently operating in the Northern hemisphere. The experiment aims to detect high-energy neutrinos up to $10^{4} \mathrm{TeV}$ using a 3-dimensional array of 885 photomultipliers distributed in 25 storeys along 12 vertical lines. The detection is based on the measurement of Cherenkov light emitted by charged leptons resulting from charged-current neutrino interactions in the matter surrounding the telescope. The accurate measurements of the photon arrival times and of the deposited charge together with a precise knowledge of the actual positions and orientations of the photo sensors allow the reconstruction of the direction of neutrinos with good angular resolution (about $0.3^{\circ}$ for muon neutrinos above a few $\mathrm{TeV}$ ) and of their energy. ANTARES is performing an indirect search for dark matter by looking for a statistical excess of neutrinos coming from astrophysical massive objects, such as the Sun, the Earth and the Galactic Centre. This excess could be an evidence of the possible annihilation of dark matter particles in the centre of these objects. In the most accepted scenario, the dark matter is composed by WIMP particles. These particles can be scattered by the nuclei of these astrophysical bodies and get gravitationally trapped, accumulating in their inner core. Here they can interact with other WIMPs, in self-annihilation reactions, producing some standard model particles that, in subsequent steps, originate neutrinos that can be detected at Earth. The preliminary results of the sensitivity of the ANTARES neutrino telescope to the indirect detection of dark matter fluxes will be presented for different dark matter models.
\end{abstract}

\section{Introduction}

\subsection{Dark Matter}

The energetic balance of the different components of the Universe has been verified, in the recent period, by many measurements based on the current abundance of cosmological data: the WMAP satellite observations[1], the studies of Ia supernovae[2] among others. These experiments provide the confirmation that only the $26 \%$ of the energy balance of the Universe is under the form of matter

a e-mail: paolo.fermani@ roma1.infn.it

This is an Open Access article distributed under the terms of the Creative Commons Attribution License 2.0, which permits unrestricted use, distribution, and reproduction in any medium, provided the original work is properly cited. 
( $\Omega_{m}=0.26$ ), with a baryonic component of only $\Omega_{b}=0.044$. This means that the $81 \%$ of matter in our Universe has a non baryonic nature $\left(\Omega_{c d m}=0.21\right)$. This kind of matter, that we can not see directly, has been called Dark Matter (DM) since it is not luminous. However this 'dark' component is necessary to explain astrophysical phenomena such as the rotational curves in spiral galaxies[3] and the Bullet cluster merging[4]. To explain these behaviours of astrophysical objects several models and DM candidates were proposed in last decades. Basically the candidate searched to be the components of Dark Matter must have the following characteristics: they must be massive, neutral, stable on cosmological time scales and only weakly and gravitationally interacting. In principle neutrinos can satisfy these requirements. But, since they have relativistic velocities they can not be interpreted as possible DM candidates because they were not able to form the structures which can be seen in our Universe. Thus, there are no Standard Model particles sharing these properties. The previous listed characteristics can be reproduced in several models[5]. One of the most popular and tested scenario is that of Dark Matter composed of the Weakly Interacting Massive Particles (WIMPs). In this paper we focus our attention on two of the most popular models: Supersymmetry (SUSY)[6] and Universal Extra Dimensions (UED), in particular their constrained versions: CMSSM and mUED, where the WIMP particles (lightest neutralino and lightest Kaluza-Klein particle) are stable due to the conservation of R-parity and of KK-parity respectively. The WIMPs of our galactic halo can be experimentally detected in two different ways[7]. The first is the direct search, which aim is to detect the recoil energy of the target nuclei impacted by the WIMP; the second is the indirect search, based on the detection of the products of WIMP annihilations in massive celestial bodies (stars, planets, galaxies). WIMPs loose their energy through consecutive elastic scattering on nuclei and can be gravitationally captured in massive objects like the Sun, where they reach the inner core and there they can self-annihilate producing some Standard Model particles. The interaction producing directly neutrinos (of the same energy of the WIMP mass) is suppressed for non relativistic particles in the CMSSM case and permitted in the mUED one. These SM particles eventually decay producing neutrinos that can be observed at Earth with large neutrino telescopes such as ANTARES or IceCube[8].

\subsection{Neutrino telescopes and detection principle}

In recent years several neutrino telescopes have been built to perform neutrino astronomy. This kind of telescopes are based on the detection of the Cherenkov light induced by the propagation of relativistic muons generated by neutrino interactions in transparent media like water. Since the neutrino cross-section has a small value and the fact that the neutrino flux decreases with the increasing energy, detectors of large mass are necessary. In this article we present the results of the ANTARES experiment. Since the Earth acts as a shield against all the particles except neutrinos, a neutrino telescope mainly uses the detection of up-going muons as a signature of a muon neutrino $\left(v_{\mu}\right)$ interaction in the Earth below the detector. The muon, travelling in water, induces Cherenkov light that can be detected by the optical modules. The wide range of distance covered by muons, permit to observe also interactions that happened hundreds meters far from the detector. Neutrinos of different flavours can also be detected, but with less efficiency and angular precision because the travelled distances of the respective leptons are short. ANTARES is able to detect muons with a minimum energy of $20 \mathrm{GeV}$ and also the neutrino direction with an accuracy of roughly $0.3^{\circ}$ for energies beyond $10 \mathrm{TeV}$.

\subsection{The ANTARES detector}

ANTARES is the first and largest submarine neutrino telescope in the Northern hemisphere[9]. It was completed in 2008 and located $\left(42^{\circ} 48^{\prime} \mathrm{N}, 6^{\circ} 10^{\prime} \mathrm{E}\right)$ in the Mediterranean Sea at roughly $2475 \mathrm{~m}$ 
depth, $42 \mathrm{~km}$ offshore of the cost of Toulon (France). The detector consists of a three-dimensional array of $88510 "$ PMTs disposed in 12 vertical strings. These strings are spread over an area of about $0.1 \mathrm{~km}^{2}$. The basic unit of the detector is the Optical Module (OM), containing one PMT and the associated electronics[10], housed in a pressure resistant glass sphere with a mu-metal cage to minimize the effect of the Earth's magnetic field. The OMs are grouped together in 25 storeys (of three $\mathrm{OMs}$ ) for each string interconnected via an electro-mechanical cable with the exception of one string which has 20 storeys since the last five are devoted to acoustic measurements. The OMs are arranged with the axis of the PMT tubes $45^{\circ}$ below the horizontal plane in order to increase the efficiency to detect up-going events. The height of the instrumented strings is $300 \mathrm{~m}$. The distance between two consecutive storeys is $14.5 \mathrm{~m}$. The horizontal distance between two adjacent strings is $60-75 \mathrm{~m}$. There is also one instrumented line for sea environmental studies. The top of the string consists of a buoy and they are anchored on the sea bed. The absolute position of the detector components as a function of time is obtained through an acoustic triangulation system combined with an orientation system that permits to determine the inclination and orientation of the single storeys[11]. The absolute UTC time accuracy is guaranteed by a GPS system and by the $25 \mathrm{MHz}$ clock of the detector.

\section{Dark Matter indirect search towards the Sun}

Here we report the analysis performed to search for a signal towards the Sun with the data taken by the ANTARES experiment in the period 2007-2008. The Sun is a very interesting source for Dark Matter search. The identification of a possible signal would have a crucial importance: we do not expect such a kind of signal arising from the Sun, since the solar neutrinos have a lower energy ${ }^{1}$. Other analyses with the same data sample performed in the collaboration are [12-14].
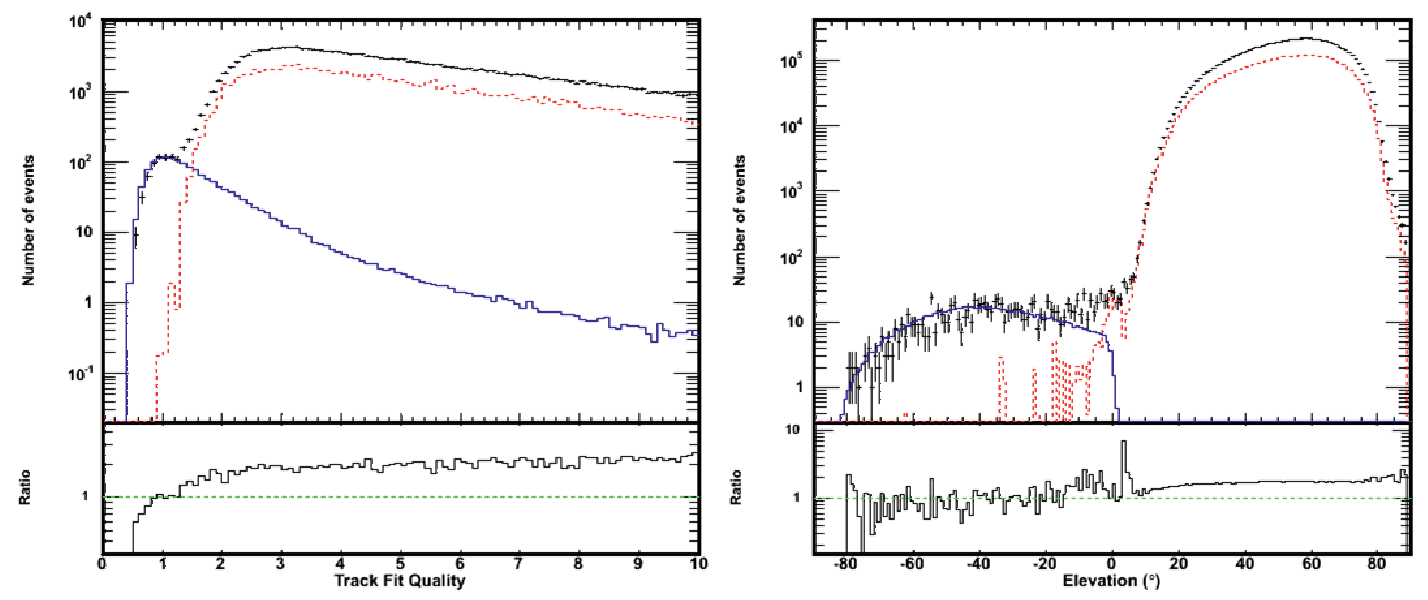

Figure 1. Comparison between data and simulation for the track fit quality (left) and the elevation coordinate of the events (right). A $Q<1.4$ is applied. The red dashed line represents the simulated atmospheric muons; the blue pointed line shows the simulated atmospheric up-going neutrinos; the black crosses represent scrambled data. The ratio of data over the simulation is shown below the main plot.

\footnotetext{
${ }^{1}$ The neutrinos produced in the Sun's corona by cosmic rays are negligible
} 


\subsection{Data and Monte-Carlo simulations}

During the large part of the year 2007 ANTARES was in a 5-lines configuration (185.5 days of active detector); while in the year 2008 the detector configuration varied: 10,9,12 lines (189.8 days of active detector). The first task in the ANTARES analyses is the discrimination between signal and background events. The main two background sources are the large flux of atmospheric muons and the flux atmospheric neutrinos both produced in the interaction of cosmic rays in the Earth's atmosphere. In order to reduce the first kind of background, the detector is installed at large depth. Moreover, only upgoing events are accepted. Still, a small fraction of atmospheric muons are reconstructed as upgoing. By imposing strict quality cuts in the tracks, they can be further removed. The second background is irreducible since neutrinos can pass trough the Earth detected as up-going events; but we can consider that atmospheric neutrinos are isotropically distributed all over the sky while the signal neutrinos are expected to peak in the Sun direction only: we are looking for an excess of events over an expected background. In figure 1 an example of data-Monte Carlo comparison where the two kinds of backgrounds are involved it is shown. For the background estimation we used the scrambled data $^{2}$ (see figure 2) to reduce the effects of the possible systematic uncertainties. To reconstruct both data and Monte Carlo events a fast and reliable algorithm, called BBFit, has been developed in the ANTARES collaboration[15]. This algorithm is based on the multiple coincidences of the Cherenkov photons arriving on the OMs of the apparatus (hits). Then, the minimization of a $\chi^{2}$ function, containing the difference between expected and measured hits, permits to reconstruct the track of the events with a given quality $Q$. The simulation of signal events from Dark Matter annihilation in the
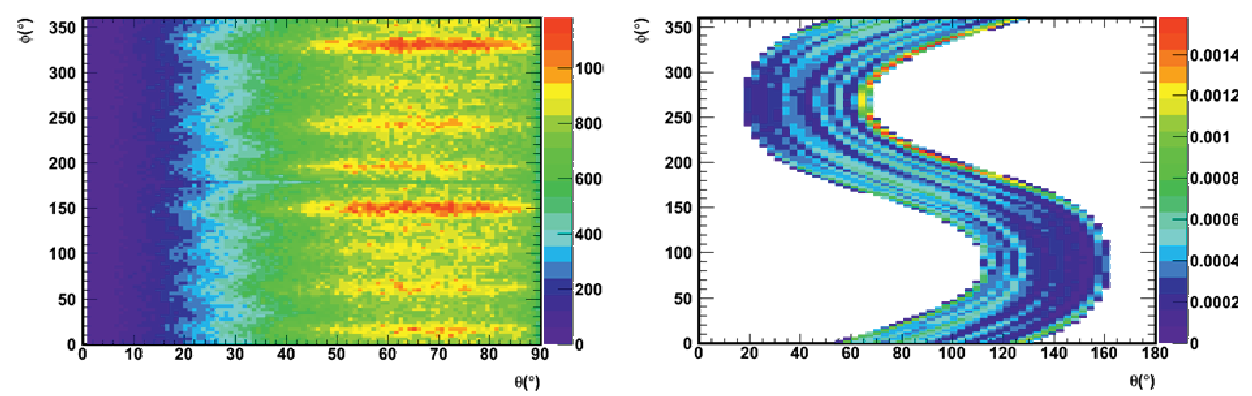

Figure 2. Distribution of up-going background events (scrambled data) in the local coordinate system (left) and an example of the Sun visibility (on the ANTARES location) in the period of data taking 2007-2008 in the same coordinates (right).

Sun is computed with the WimpSim package[16], which can evaluate the differential neutrino spectra for several annihilation channels and different WIMP masses. The neutrino interactions in the Sun medium, the regeneration of $\tau$ leptons together with the standard neutrino oscillation scenario have been taken into account. We assume also that capture and annihilation rates are in equilibrium in the Sun. For the CMSSM scenario, we have used as benchmark annihilation channels $W^{+} W^{-}, \tau^{+} \tau^{-}$and $b \bar{b}$. For mUED, we have used $c \bar{c}, \tau^{+} \tau^{-}, b \bar{b}, t \bar{t}$ and $v \bar{v}$. We have to note that we can consider the Sun as a point source, since the annihilation reactions happens in its core.

\footnotetext{
${ }^{2}$ Scrambled data are obtained randomising the UTC time of the events in the considered data taking period
} 


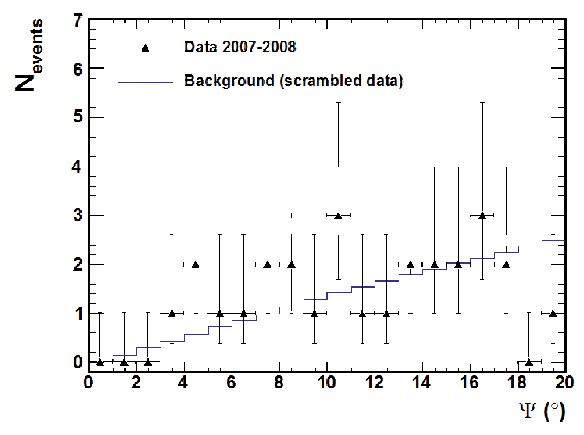

Figure 3. Distribution of the spatial angle $\Psi \in\left[0^{\circ}, 20^{\circ}\right]$ of the event tracks with respect to the Sun's direction for the expected background computed from the time-scrambled data (solid blue line) compared to the data after the basic selection criteria (black triangles). A $1 \sigma$ Poisson uncertainty is shown for each data point (black crosses).

\subsection{Event selection optimization}

In the analysis a binned search has been done: it means to count the number of events (signal and background) within an angular bin of given aperture $\Psi$ centred in the Sun position (see figure 3). It is important to note that a blind ${ }^{3}$ procedure has been followed to choose the cuts to apply to data. The optimization of these cuts has been performed using the Model Rejection Factor (MRF) method[17]. Two parameters were considered for the optimization: the quality of the event reconstruction $Q$ and the half-cone angle aperture around the Sun $\Psi$. The MRF gives the optimized set of these two parameters to obtain the best average upper limit (at $90 \%$ C.L.) on the flux of neutrinos ${ }^{4}$ deriving from Dark Matter annihilation in the Sun. This average flux upper limit can be expressed by:

$$
\bar{\phi}_{v}^{90 \%}=\frac{\bar{\mu}^{90 \%}}{A_{e f f}\left(M_{\text {WIMP }}\right) \times T_{e f f}}
$$

where $\bar{\mu}^{90 \%}$ is the average upper limit in the event number, derived from the Feldman and Cousins calculations[18], $\mathrm{A}_{e f f}\left(\mathrm{M}_{\text {WIMP }}\right)$ is the effective area and $\mathrm{T}_{\text {eff }}$ is the active detector data taking period. This evaluation has been done for each mass and channel of the two models chosen.

\section{Results and conclusions}

Using equation 1 it is possible to evaluate the sensitivity for all the Dark Matter models considered. In figure 4 the average upper limits in the neutrino flux as a function of the WIMP mass are shown. The different channels are separate since there is a high dependence of branching ratios over the CMSSM parameter space; this is not in the mUED case, so it is possible to combine the channels conserving a reliable representation. The best limits in CMSSM arise from the $W^{+} W^{-}$and $\tau^{+} \tau^{-}$channels since they have a hard energy spectrum. In the mUED case the channel that most contribute is the $\tau^{+} \tau^{-}$: the total sensitivity is close to the one of that channel of the CMSSM case. It is also possible to pass

\footnotetext{
${ }^{3}$ In this way the cuts are selected before to look at the source of interest, avoiding a possible bias.

${ }^{4}$ here neutrinos stands for the sum of neutrinos and anti-neutrinos.
} 


\section{EPJ Web of Conferences}

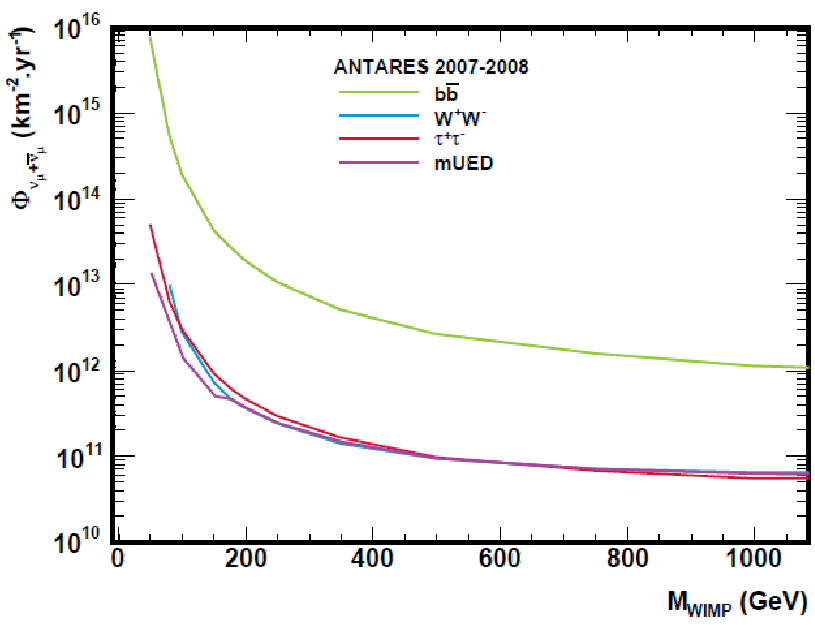

Figure 4. $90 \%$ C.L. upper limit on the neutrino plus anti-neutrino flux as a function of the WIMP mass in the range $M_{W I M P} \in[10 \mathrm{GeV} ; 1 \mathrm{TeV}]$ for the three channels $b \bar{b}, W^{+} W^{-}, \tau^{+} \tau^{-}(C M S S M)$ and a combination of channels (mUED).

from these limits on neutrino fluxes to the limits on the spin-dependent cross-section of the WIMPs with protons $\sigma_{H, S D}$. The differential neutrino flux is:

$$
\frac{d \phi_{v}}{d E_{v}}=\frac{\Gamma}{4 \pi d^{2}} \sum_{f} B_{f}\left(\frac{d N_{v}}{d E_{v}}\right)_{f}
$$

where $d$ is the distance between the Sun and the Earth, $\left(d N_{v} / d E_{v}\right)_{f}$ is the differential number of neutrino events for each channel, $B_{f}$ the relative branching ratios and $\Gamma \simeq C_{\otimes} / 2$ is the annihilation rate as related to the capture rate $C_{\otimes}$ assuming the equilibrium of the two rates inside the $\mathrm{Sun}^{5}$. In figure 5 the limits on the $\sigma_{H, S D}$ values (obtained with a scan of the SuperBayes package[19]) for CMSSM and mUED models can be seen. In the spin-dependent case both ANTARES and IceCube limits are better compared with the direct search experiments (this is not in the case for limits on the spin-independent cross-section). Summarising, the indirect search for Dark Matter is a main goal for the large neutrino telescopes like ANTARES, it also provide a complementarity with the direct search. In the Sun analysis with 2007-2008 data presented in this article, the detector efficiency for two common Dark Matter models: CMSSM and mUED has been computed, evaluating the background in the Sun direction in a two dimensional parameter space. Finally the sensitivity has been evaluated also in the spin dependent case for the same models.

\footnotetext{
${ }^{5}$ The capture rate expression, assuming a Navarro Frank and White (NFW) profile for the Dark Matter density and a Maxwell-Boltzmann velocity distribution, is: $C_{\otimes} \simeq 3.35 \times 10^{18} \mathrm{~s}^{-1} \times\left(\frac{\rho_{\text {local }}}{0.3 \mathrm{GeVcm}^{-3}}\right) \times\left(\frac{270 \mathrm{kms}^{-1}}{v_{\text {local }}}\right) \times\left(\frac{\sigma_{H, S D}}{10^{-6} \mathrm{pb}}\right) \times\left(\frac{T e V}{M_{W I M P}}\right)^{2}$.
} 

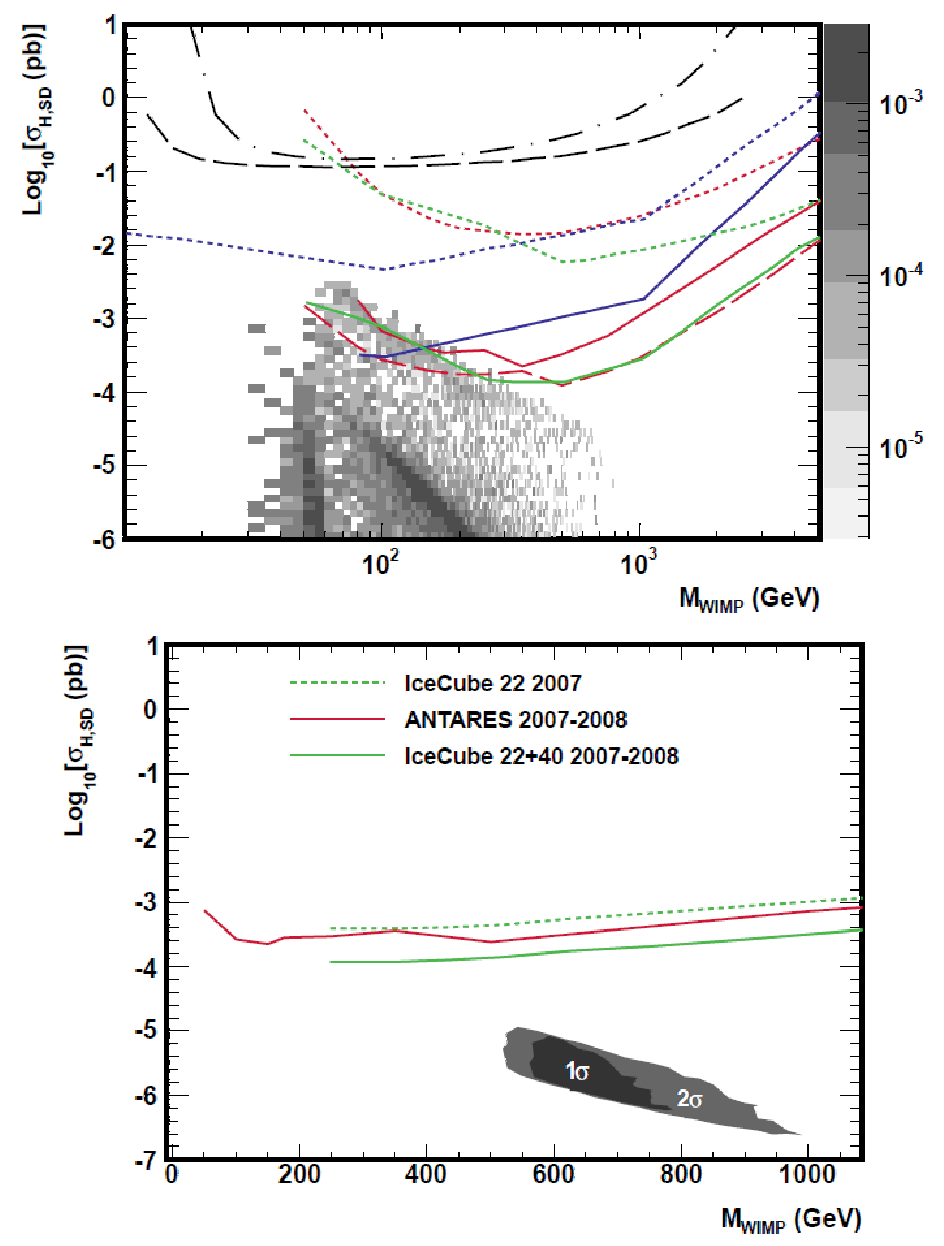

Figure 5. up) $90 \%$ C.L. upper limits on the spin-dependent WIMP-proton cross-section as a function of the WIMP mass in the range $M_{\text {WIMP }} \in[10 \mathrm{GeV} ; 5 \mathrm{TeV}]$, for the three channels: $b \bar{b}$ (dotted line), $W^{+} W^{-}$(solid line), $\tau^{+} \tau^{-}$(dashed line), for ANTARES (red line) compared to the results of other indirect search experiments: SuperKamiokande 1996 - 2008 (blue line) and IceCube-40 plus AMANDA 2001 - 2008 (green line) and the results of direct search experiments: KIMS 2007 (black dot-dashed line) and COUPP 2011 (black dashed line); down) The same plot for a combination of channels in the mUED model (red line). Results from IceCube-22 2007 (green dotted line) and IceCube-22+40 2007 - 2008 (green solid line) are shown for comparison.

\section{References}

[1] G. Hinshaw et al, WMAP collaboration, Five-Year Wilkinson Microwave Anisotropy Probe Observations: data processing, sky maps, and basic results, Astrophys. J. Supp. 180, 225-245 (2009).

[2] M. Kowalski et al, Improved cosmological constraints from new, old and combined supernova data set, Astrophys. J. 686, 749-778 (2008). 
[3] T.S. Van Albada et al, Distribution of dark matter in the spiral galaxy NGC 3198, Astrophys. J. 395, 305 (1985).

[4] D. Clowe et al, A direct empirical proof of the existence of dark matter. Astrophys. J. 648, 109, (2006).

[5] G. Bertone, D. Hooper, J. Silk, Particle dark matter: evidence, candidates and constraints, Phys. Rept. 405, 279 (2005).

[6] G. Jungman, M. Kamionkowski, K. Griest, Supersymmetric dark matter, Phys. Rep. 267, 195 (1996).

[7] Y. Mambrini, C. Muñoz, A comparison between direct and indirect dark matter search, JCAP 410, 3 (2004).

[8] A. Achterberg et al, IceCube Collaboration, First Year Performance of the IceCube Neutrino Telescope, Astrop. Phys. 26, 155-173 (2006).

[9] M. Ageron et al, ANTARES Collaboration, ANTARES: The first undersea neutrino telescope, Nucl. Instrum. Meth. A 656, 11-38 (2011).

[10] J.A. Aguilar et al, ANTARES Collaboration, Performance of the front-end electronics of the ANTARES Neutrino Telescope, Nucl. Instrum. Meth. A 622, 59-73 (2010).

[11] S. Adrian-Martinez et al, ANTARES Collaboration, The positioning system of the ANTARES neutrino telescope, JINST 7, T08002 (2012).

[12] S. Adrian-Martinez et al, ANTARES Collaboration, First search for point sources of high energy cosmic neutrinos with the ANTARES neutrino telescope, Astrophys. J. Lett. 743, L14-L19 (2011).

[13] S. Adrian-Martinez et al, ANTARES Collaboration, Search for relativistic magnetic monopoles with the ANTARES neutrino telescope, Astropart. Phys. 35, 634-640 (2012).

[14] S. Adrian-Martinez et al, ANTARES Collaboration, Measurement of atmospheric neutrino oscillations with the ANTARES neutrino telescope, Phys. Lett. B 714, 224-230 (2012).

[15] J.A. Aguilar et al, ANTARES Collaboration, A fast algorithm for muon track reconstruction and its application to the ANTARES neutrino telescope, Astropart. Phys. 34, 652-662 (2011).

[16] J. Edsjö, WimpSim Neutrino Monte Carlo, http://www.fysik.su.se/ edsjo/wimpsim/.

[17] G.C. Hill, K. Rawlins, Unbiased cut selection for optimal upper limits in neutrino detectors: the model rejection potential technique, Astropart.Phys. 19, 393-402 (2002).

[18] G.J. Feldman, R.D. Cousins, Unified approach to the classical statistical analysis of small signals, Phys.Rev. D57, 3873-3889 (1999).

[19] F. Feroz et al., Challenges of Profile Likelihood Evaluation in Multi-Dimensional SUSY Scans, JHEP 1106, 042 (2011). 